\title{
INTER- AND INTRAGRAIN MAGNETIZATION OF THALLIUM-BASED SUPERCONDUCTORS
}

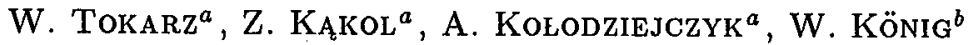 \\ AND G. GRITZNER ${ }^{b}$ \\ ${ }^{a}$ Department of Solid State Physics, Faculty of Physics and Nuclear Techniques \\ University of Mining and Metallurgy, Al. Mickiewicza 30, 30-059 Kraków, Poland \\ ${ }^{b}$ Institut für Chemische Technologie Anorganischer Stoffe \\ Johannes Kepler Universität Linz, 4040 Linz, Austria
}

\begin{abstract}
We report on magnetization and magnetic hysteresis measurements for the $\left(\mathrm{Tl}_{0.6} \mathrm{Bi}_{0.5}\right)\left(\mathrm{Sr}_{0.9} \mathrm{Ba}_{0.1}\right)_{2} \mathrm{Ca}_{2} \mathrm{Cu}_{3} \mathrm{O}_{y} \mathrm{Ag}$-sheathed superconducting tape as well as for the powdered and bulk $\left(\mathrm{Tl}_{0.6} \mathrm{~Pb}_{0.5}\right)\left(\mathrm{Sr}_{0.9} \mathrm{Ba}_{0.1}\right)_{2} \mathrm{Ca}_{2} \mathrm{Cu}_{3} \mathrm{O}_{y}$ superconductors. For the investigated tape and bulk specimens the low field magnetization showed a deviation from the ideal linear $M(H)$ dependence, which was attributed to the superposition of inter- and intragrain superconducting currents. Fitting of the theoretical magnetization curves to the experimental data allowed for the independent determination of the intragrain critical current densities for the superconducting grains and intergrain critical current densities for the weak link system at $77 \mathrm{~K}$.
\end{abstract}

PACS numbers: 74.72.-h, 74.72.Fq, 74.50.+r

\section{Introduction}

Elevated transition temperature $T_{c}$, high critical currents and high reversible field $H_{\mathrm{r}}$ are crucial parameters for technical applications of superconducting materials. All these values can be evaluated directly from magnetization and hysteresis loops measurements together with many microscopic parameters such as the coherence length and the penetration depth. In the ceramic specimens, due to their granular structure, description of the magnetization process involves superposition of magnetization of the intergranular (weak links between superconducting grains) and intragranular regions (superconducting grains). Thus it is of great importance to estimate the influence of samples microstructure on the magnetization process. For the purpose of such analysis we have selected samples with different porosity and different grain alignment i.e. powdered sample, sintered ceramics and well aligned Ag-sheathed tape. Tl-based specimens were chosen because of their high transition temperature and high reversible field. 


\section{Experimental results and discussion}

A sintered ceramic $\left(\mathrm{Tl}_{0.6} \mathrm{~Pb}_{0.5}\right)\left(\mathrm{Sr}_{0.9} \mathrm{Ba}_{0.1}\right)_{2} \mathrm{Ca}_{2} \mathrm{Cu}_{3} \mathrm{O}_{y}$ superconductor with a critical temperature $\left(T_{c(0)}\right)$ of $118 \mathrm{~K}$ was prepared with $T_{c}$ of $118 \mathrm{~K}$ by a procedure described elsewhere [1-3]. The porosity of this sample was $9 \%$ and the amount of the secondary phase was $5 \%$. Part of that sample was ground into powder. The second sample, an $\mathrm{Ag}$-sheathed tape of $\left(\mathrm{Tl}_{0.6} \mathrm{Bi}_{0.16} \mathrm{~Pb}_{0.24}\right)_{2}\left(\mathrm{Sr}_{0.9} \mathrm{Ba}_{0.1}\right)_{2} \mathrm{Ca}_{2} \mathrm{Cu}_{3} \mathrm{O}_{y}$ with a $T_{\mathrm{c}}$ of $118 \mathrm{~K}$ was fabricated from precursors prepared either by the spray-drying of nitrates or by the tartrate gel technique [3]. Following the addition of $\mathrm{Tl}_{2} \mathrm{O}_{3}, \mathrm{PbO}$, and $\mathrm{Bi}_{2} \mathrm{O}_{3}$, the powders were compacted and sintered into superconducting pellets. The resultant pellets were milled, filled in silver tubes drawn to wire and then rolled to $0.25 \times 2 \mathrm{~mm}$ tapes. Final sintering was carried out in air at $850^{\circ} \mathrm{C}$ for $0.5 \mathrm{~h}$ followed by annealing at $750^{\circ} \mathrm{C}$ for $50 \mathrm{~h}$. The filling factor of the superconductor in the wire was $40 \%$, the porosity of the superconductors was $18 \%$ and the amount of secondary phases $2 \%$. The degree of grain alignment in the tape was estimated to about $95 \%$.

Magnetization measurements were carried out in magnetic fields up to $1 \mathrm{~T}$ with the use of a standard vibrating magnetometer at the temperature $77 \mathrm{~K}$. The zero field cooling procedure (ZFC) was applied. Magnetization of the tape was measured with the magnetic field applied parallell and perpendicular to the length of the tape i.e. in the direction of alignment of the grains. Magnetization curves and hysteresis loops for all samples are presented in Fig. 1a, whereas the details of the low field magnetization are shown in Fig. 1b.

The deviation from the ideal, low field linear $M(H)$ dependence found for the ceramic and the tape-shaped samples was not observed for the powdered sample. This indicates that the intergranular regions play a significant role in the magnetization processes and must be taken into account. Therefore we followed the model by Mahel and Pivarc [4] in analysing the magnetization data. In this model the high- $T_{\mathrm{c}}$ superconductor ceramics are treated as superconducting domains (grains) embedded in a quasi-continuous weakly superconducting intergranular medium. Both media were treated as hard type II superconductors. The specimen magnetization $M_{\mathrm{s}}$ is considered to contain the magnetization $M_{\mathrm{g}}$ of grains (of average size) and the magnetization $M_{\mathrm{i}}$ of the intergranular host averaged over volume: $M_{\mathrm{s}}=M_{\mathrm{i}}+f \nu M_{\mathrm{g}}$. Here $\nu$ is the part of the sample volume occupied by the grains and $f$ is a portion of the magnetic flux produced by intragranular currents registered in the experiment. The $(1-f)$ portion remains confined in the intergranular regions within the sample. The field experienced by the grains $H_{\mathrm{i}}$ can be consequently written as

$$
H_{\mathrm{i}}=H+M_{\mathrm{i}}\left(1-N_{\mathrm{s}}\right)-M_{\mathrm{g}} \nu\left[1-f\left(1-N_{\mathrm{s}}\right)\right]
$$

and magnetic field $H_{\mathrm{s}}$ near the sample surface as

$$
H_{\mathrm{s}}=H-N_{\mathrm{s}} M_{\mathrm{s}} \text {, }
$$

where $N_{\mathrm{s}}$ is the sample demagnetization factor and $H$ is applied external magnetic field. The equilibrium magnetization for both inter- $M_{\mathrm{i}}$ and intragranular media $M_{\mathrm{g}}$ were modeled by the extended Kim-Anderson model [5-7] with the 


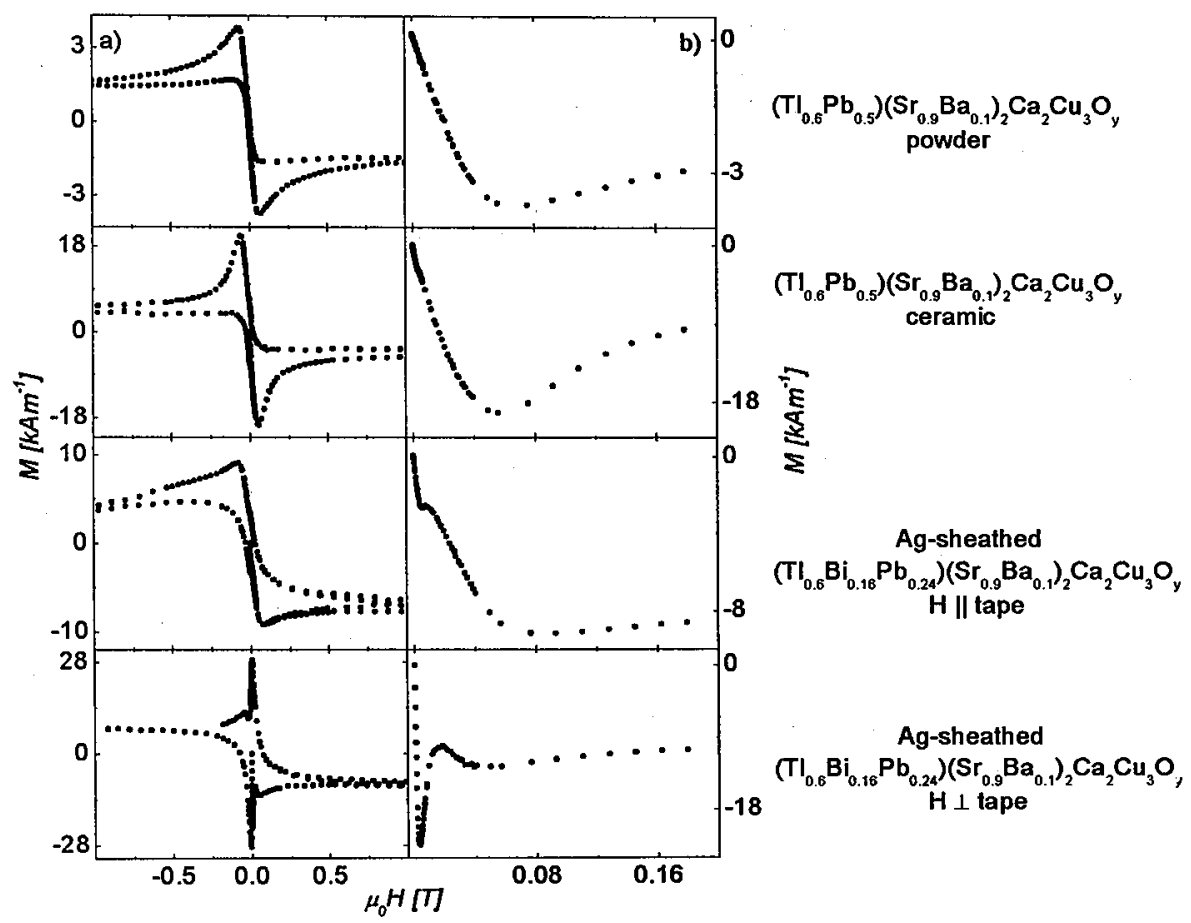

Fig. 1. (a) Hysteresis loops and (b) low field initial magnetization for the investigated samples.

field dependent critical current density

$$
J_{\mathrm{c}}=\frac{k}{B_{0}+\left|\mu_{0} H\right|},
$$

where $k$ and $B_{0}$ are constant. Here $H$ denotes $H_{\mathrm{i}}$ and $H_{\mathbf{s}}$ respectively for different media. The average flux density inside the specimen in ZFC process can be then expressed as

$$
B(H)=G(a)-G\left(x_{0}\right)-\frac{B_{0}}{a^{2}}\left(a^{2}-x_{0}^{2}\right) .
$$

For intergranular medium $a$ represents the thickness of the sample and for intragranular medium $a$ is the average grain size; $x_{0}=a-\left[\left(B_{0}+\mu_{0} H\right)^{2}-B_{0}^{2}\right] /\left(2 \mu_{0} k\right)$ is the penetration area of magnetic field. The function $G(x)$ is defined as $G(x)=$ $\left(\mu_{0} k a\right)^{-2}(3 p a-2 q)(p x+q)^{3 / 2} / 15$, where $p=2 \mu_{0} k, q=\left(B_{0}+\mu_{0} H\right)^{2}-2 \mu_{0} k a$. Finally, the magnetization can be calculated from general formula

$$
M(H)=\frac{B(H)}{\mu_{0}}-H
$$

with $M=M_{\mathrm{i}}, H=H_{\mathrm{s}}$ for intergranular regions and with $M=M_{\mathrm{g}}, H=H_{\mathrm{i}}$ for intragranular area. Typical fitted magnetization curves are presented in Fig. 2.

Based on these data the critical currents for grains and weak links at $H=0$ and at $T=77 \mathrm{~K}$ were calculated. The obtained data are collected in Table. 


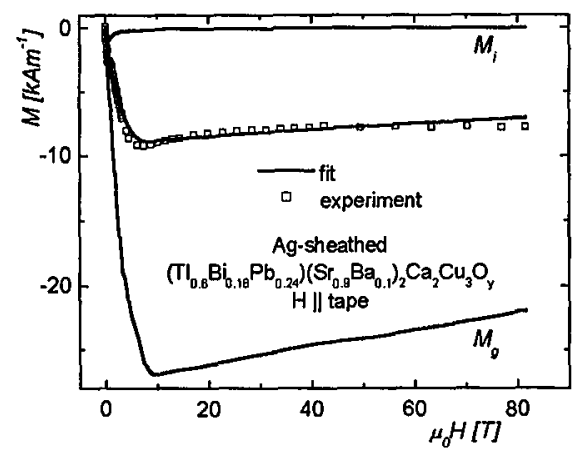

Fig. 2. Results of fitting to the magnetization as indicated.

TABLE

Critical currents for weak links $J_{\mathrm{cJ}}$ and grains $J_{\mathrm{cg}}$.

\begin{tabular}{l|r|c}
\hline \hline & $J_{\mathrm{cJ}}\left[\mathrm{A} \mathrm{m}^{-2}\right]$ & $J_{c \mathrm{~g}}\left[\mathrm{~A} \mathrm{~m}^{-2}\right]$ \\
\hline$\left(\mathrm{Tl}_{0.6} \mathrm{~Pb}_{0.5}\right)\left(\mathrm{Sr}_{0.9} \mathrm{Ba}_{0.1}\right)_{2} \mathrm{Ca}_{2} \mathrm{Cu}_{3} \mathrm{O}_{y}$ & $1.1 \times 10^{7}$ & $3.0 \times 10^{10}$ \\
$\left(\mathrm{Tl}_{0.6} \mathrm{Bi}_{0.16} \mathrm{~Pb}_{0.24}\right)_{2}\left(\mathrm{Sr}_{0.9} \mathrm{Ba}_{0.1}\right)_{2} \mathrm{Ca}_{2} \mathrm{Cu}_{3} \mathrm{O}_{y}, H \|$ tape & $13 \times 10^{7}$ & $5.4 \times 10^{10}$ \\
$\left(\mathrm{Tl}_{0.6} \mathrm{Bi}_{0.16} \mathrm{~Pb}_{0.24}\right)_{2}\left(\mathrm{Sr}_{0.9} \mathrm{Ba}_{0.1}\right)_{2} \mathrm{Ca}_{2} \mathrm{Cu}_{3} \mathrm{O}_{y}, H \perp$ tape & $60 \times 10^{7}$ & $3.9 \times 10^{10}$
\end{tabular}

\section{Conclusion}

The observed anomalies in the initial magnetization for solid superconductors investigated in this work suggest that intergranular weak links play important role in high- $T_{\mathrm{c}}$ ceramic superconductors. This is reflected in high critical currents for tape-shaped specimens characterized by well formed intergranular links.

\section{Acknowledgment}

This work was supported by the Committee for Scientific Research (Poland) under the grant No. 2P03B14717, by the Faculty of Physics and Nuclear Techniques, University of Mining and Metallurgy, Kraków, the Fonds zur Förderung der Wissentschaftlichen Forschung (Austria, project P 13945-TEC), and by the Österreichischer Akademischer Austauschdienst, Wissenschaftlich-technische Zusammenarbeit mit Polen (project 3/99).

\section{References}

[1] W. König, G. Gritzner, M. Reissner, W. Steiner, Physica C 258, 175 (1996).

[2] M. Mair, W. König, G. Gritzner, Supercond. Sci. Technol. 8, 894 (1995).

[3] W. König, G. Gritzner, P. Diko, J. Kovác, M. Timko, J. Mater. Chem. 5, 879 (1995); W. König, M. Mair, G. Gritzner, J. Supercond. 11, 107 (1998).

[4] M. Mahel, J. Pivarč, Physica C 308, 147 (1998).

[5] Y.B. Kim, C.F. Hempstead, A.R. Strnad, Phys. Rev. Lett. 9, 306 (1962).

[6] Y.B. Kim, C.F. Hempstead, A.R. Strnad, Phys. Rev. 129, 528 (1963).

[7] K. Yamamoto, H. Mazaki, H. Yasuoka, Phys. Rev. B 47, 915 (1993). 